\title{
Respuesta fisiológica del cultivo de remolacha (Beta vulgaris L.) bajo condiciones de un sustrato salino en Madrid, Cundinamarca
}

\author{
César Pérez ${ }^{1}$, Paola Andrea Piza², Ángela Patricia Salamanca²
}

Recibido: noviembre 1 de 2011 Aprobado: marzo 31 de 2012

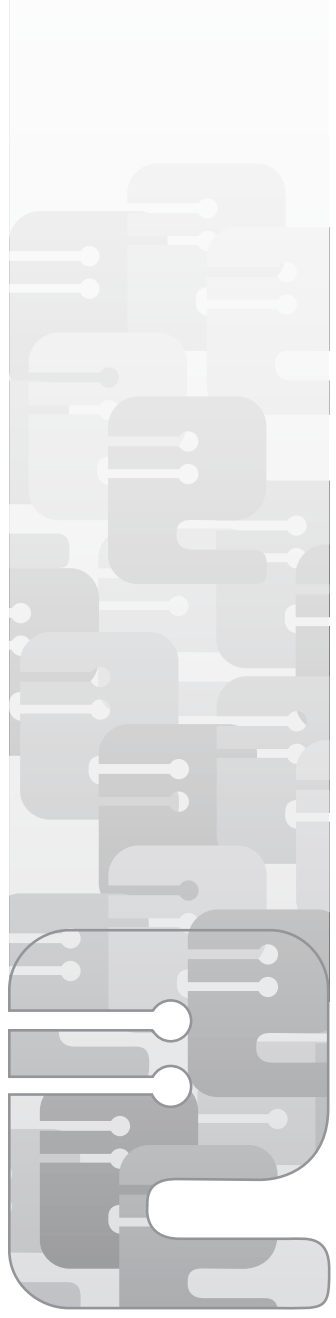

Resumen

El presente artículo recoge los resultados de la investigación relacionada con la respuesta fisiológica del cultivo de remolacha (Beta vulgaris L.) bajo condiciones de estrés salino, realizada en el Centro Agroecológico de Investigación y Capacitación -Ceagro- San Pablo, ubicado el municipio Madrid (Cundinamarca). Para llevar a cabo el objetivo propuesto, se analizaron los siguientes parámetros: peso turgente, peso fresco, peso seco y contenido hídrico relativo. Se estableció un cultivo de remolacha (Beta vulgaris $L$ ) característico por ser un cultivo halófito, tolerante a sustratos salinos. Se realizaron dos tratamientos en el cultivo: tratamiento convencional y agroecológico, donde se obtuvieron muestras in situ a los 45, 60 y 120 días respectivamente, después de haber sembrado las plántulas. La distribución se ejecutó en diez camas, en un área de $250 \mathrm{~m} 2$, generando una densidad de siembra total de 2.100 plantas. Los resultados arrojados por la investigación expresan un contenido hídrico relativo en los dos tratamientos de 83\% y 69\% para el tratamiento agroecológico y convencional, respectivamente; bajo condiciones de salinidad. Estadísticamente, el mejor comportamiento ante la condición salina se evidenció en el tratamiento agroecológico, lo cual evidencia la tolerancia del cultivo de remolacha en condiciones salinas de $4.23 \mathrm{dS} \mathrm{m}$-1, mostrando valores de respuesta fisiológica (CHR) mayor a los resultados del tratamiento convencional y contribuyendo a minimizar el deterioro de la capa cultivable.

Palabras Clave: Respuesta Fisiológica, Remolacha, Salinidad, Contenido Hídrico Relativo

\begin{abstract}
The paper presents the reserch results related to the physiological response of beet (Beta vulgaris L.) under salt stress conditions Agro-ecological Center of Research and Training-Ceagro-San Pablo, located in Madrid township (Cundinamarca). To accomplish this objective, we analyzed the following determinations: turgid weight, fresh weight, dry weight and relative water content. It was established a beet (Beta vulgaris L.), a crop being characteristic halophytic, salt tolerant substrates. Two treatments were performed in the culture; conventional treatment and agro where samples were collected in situ at 45- 60 - 120 days respectively after sowing the seedlings. The distribution was carried out in 10 beds, in an area of $250 \mathrm{m2}$, generating a total seeding plants of 2100. Results from research express relative water content in the two treatments of $83 \%$ and $69 \%$ for agro-ecological and conventional treatment, respectively, under conditions of salinity. Statistically, the best performance under saline condition was evident in agro processing, which shows the beet crop tolerance to saline conditions of $4.23 \mathrm{dS} \mathrm{m}-1$, showing values of physiological response (CHR) greater than conventional treatment outcomes and helping to minimize damage to the topsoil.
\end{abstract}

Keywords: Physiological Response, Beet, salinity, Relative Water Content

1 Estudiante de X semestre de Ingeniería Agroecológica, Corporación Universitaria Minuto de Dios -UNIMINUTO. 2 M Sc. Agroforestería M Sc. Agrobiología Ambiental. 


\section{INTRODUCCIÓN}

Químicamente el concepto relacionado con la salinidad abarca el efecto de todas las sales solubles, tanto las necesarias para la alimentación de las plantas, como las que son perjudiciales. Un suelo es considerado salino cuando tiene un exceso de sales solubles, entendiéndose como sales los diferentes componentes químicos que hacen parte de los nutrientes y requerimientos de las plantas, bien sea porque se encuentran en la solución o precipitadas en el suelo (Campos, 2009).

La aplicación indiscriminada de fertilizante es un factor determinante para la salinización de suelos en Colombia. Los nitratos son sales muy solubles, los fosfatos forman sales insolubles con el calcio y el magnesio; en suelos alcalinos el fosfato tricálcico reacciona con el carbono sódico generando fosfato sódico, que es altamente soluble y puede ser lavado, provocando deficiencias en la nutrición (Jaime et al., 2005).

Sin duda alguna, los efectos de salinización en el suelo inhiben el crecimiento de cualquier cultivo, atacando estados fenológicos de iniciación de la planta como la germinación, periodo crítico en la vida de la planta, en el que aumenta su sensibilidad a la salinidad. Dos ejemplos claros son la germinación de la cebada y el trigo, que no toleran salinidades superiores a 4-5 dS m-1 a $25{ }^{\circ} \mathrm{C}$, mientras que la remolacha, planta tolerante a la salinidad, se ve afectada si supera los $3 \mathrm{dS}$ m-1 a $25^{\circ} \mathrm{C}$ en esta etapa ( López y Acevedo, 2005).

Buscando soluciones apresuradas para combatir esta problemática se incurre en métodos como la inundación de terrenos con el fin de lavar las sales y los nutrientes, el levantamiento de camas a una altura donde el nivel freático sea controlado por esta medida; no obstante, se sabe que dicha medida es antieconómica por los costos tan elevados de la mano de obra necesaria para aplicar esta propuesta de levantamiento de camas, en grandes extensiones.

Por ello investigadores del suelo como Ortiz, Leidi y Pardo, en el año 2002, mostraron su interés por optimizar la tolerancia a la salinidad en los cultivos, empleando métodos de mejora y selección tradicionales o a través de producción de organismos modificados genéticamente. La incorporación de genes procedentes de parentales silvestres tolerantes, la domesticación de plantas halófilas silvestres y la identificación de caracteres relacionados con tolerancia empleando marcadores moleculares (Ashraf, 1994; Shannon, 1997; y, Yeo, 1998), o bien la incorporación de genes cuya expresión modifica mecanismos bioquímicos y fisiológicos involucrados en la tolerancia.
Una de las características más notables en los halófitos es la alta presión osmótica de la savia celular debido al alto contenido de sodio y cloro. Algunos halófitos aumentan progresivamente su contenido de sales y su presión osmótica durante el periodo de crecimiento, sin que las plantas sufran mayor daño. Sin embargo, en los halófitos suculentos no se registra un aumento en la presión osmótica. (Universidad Central de Venezuela, Instituto de Estudios Políticos, 1971)

\section{Salinidad en plantas}

Para extensas áreas de suelo salino en diferentes regiones climáticas, la salinidad se ha convertido en un gran problema para la producción agrícola, ya que dicha presencia de sales en la solución del suelo (particularmente en el entorno de las raíces) puede incidir en el crecimiento de las plantas por sus reacciones con el agua, efectos nutricionales y el balance de energía (Florez et al., 1996).

Royo y Cantero, en el año 1996 plantearon que la superficie agrícola del planeta afectada por salinidad y/o alcalinidad es de alrededor de mil millones de hectáreas (Szabvolcs, 1989) lo que varía, según las fuentes consultadas, desde valores de 230 millones hasta casi mil millones de hectáreas (Epstein et al., 1980), estando presente en todos los continentes. Otras estimaciones indican que un tercio de la superficie mundial de bajo riego (unas 230 millones de hectáreas) se encuentran afectadas en mayor o menor grado por la salinidad (Reeve y Fireman, 1967).

La salinidad afecta el crecimiento y producción de los cultivos al reducir el potencial hídrico de la solución del suelo, disminuyendo así la disponibilidad de agua, y al crear un desequilibrio nutritivo dado la elevada concentración de elementos $(\mathrm{Na}+, \mathrm{Cl}-)$ que pueden interferir con la nutrición mineral y el metabolismo celular. En consecuencia, los diversos efectos observados a distinta escala, desde reducción de turgencia y crecimiento hasta la pérdida de la estructura celular por desorganización de membranas e inhibición de la actividad enzimática, son el producto combinado de estrés hídrico, toxicidad iónica y desequilibrio nutricional. (Ortíz, Leidi y Pardo, 2002).

Para conseguir la adaptación a las condiciones salinas, se deben activar múltiples mecanismos: se debe aumentar la capacidad para obtener y/o retener agua y se debe restituir la homeostasis iónica. Estos mecanismos de adaptación se reflejan macroscópicamente como un menor crecimiento, modificación de la relación parte aérea/raíz y limitación de la expansión foliar, y son consecuencia de cambios bioquímicos (síntesis de ácido abscísico y solutos osmoprotectores) y fisiológicos (alteración de la permeabilidad de las membranas a los iones y al agua, cierre estomáti- 
co, disminución de transpiración y fotosíntesis, etc.). (Ortíz, Leidi y Pardo, 2002).

Una célula vegetal expuesta a un medio salino equilibra su potencial hídrico perdiendo agua, lo que produce la disminución del potencial osmótico y del potencial de turgencia. Esta situación genera señales químicas (aumento del $\mathrm{Ca}^{2+}$ libre intracelular, síntesis de $A B A$, , que desencadenan posteriores respuestas adaptativas (Hasegawa et al, 2000). Durante el proceso de ajuste se produce la acumulación de solutos orgánicos e inorgánicos que reducen el potencial osmótico celular (Wyn Jones y Gorham, 1983), y la reducción en la conductividad hidráulica de las membranas, posiblemente por disminución del número o apertura de los canales de agua (acuaporinas) (Ortíz, Leidi y Pardo, 2002).

Por lo tanto, en un suelo salino, la elevada concentración de iones $\mathrm{Na}^{+}$y Cl- (o $\mathrm{SO}_{4}^{2-}$ ) produce una interferencia en la absorción de nutrientes $\left(\mathrm{K}^{+}, \mathrm{Ca}^{2+}, \mathrm{NO}_{3}^{-}\right)$e impide la captación de los mismos, al tiempo que puede alcanzar niveles citosólicos tóxicos para el metabolismo celular. El mantenimiento del equilibrio iónico de la célula frente a los cambios del medio externo, esto es la homeostasis iónica, depende de las proteínas de membrana que regulan el flujo de iones, como las bombas de protones (ATPasas y pirofosfatasas), transportadores secundarios y canales iónicos (Niu et al., 1995; Maathuis \& Amtmann, 1999). Uno de los factores determinantes de la tolerancia celular a la salinidad reside en la capacidad de mantener una alta relación $\mathrm{K}+/ \mathrm{Na}+$ en el citosol. Un caso aparte lo constituye la entrada de aniones, que pueden interferir con la absorción de nutrientes como $\mathrm{Ca}^{2+}$ y NO$_{3}$ - e inducir deficiencias o bien presentar distinto grado de toxicidad $\left(\mathrm{Cl}^{-}\right.$o $\left.\mathrm{SO}_{4}{ }_{4}^{2-}\right)$ (Grattan \& Grieve, 1994; Ortíz, Leidi y Pardo, 2002).

Los cambios inducidos por el estrés salino en la ultra estructura, morfología y desarrollo de tejidos especializados (endodermis y exodermis) de la raíz (Harvey et al., 1985; Sánchez-Aguayo \& González-Utor, 1992; Reinhardt \& Rost, 1995) reflejan los cambios adaptativos conducentes al control de la absorción y transporte de agua y iones a la parte aérea (Ortíz, Leidi y Pardo, 2002).

El $\mathrm{K}+$ es uno de los principales solutos empleados para el ajuste osmótico en células vacuoladas o poco vacuoladas (Greenway \& Muñiz, 1980; Wyn Jones \& Gorham, 1983b). En estas últimas, el papel más importante en el ajuste osmótico lo tienen diferentes compuestos orgánicos (azúcares, aminoácidos, etc.).

\section{Contenido Hídrico Relativo - CHR}

El contenido hídrico relativo se expresa como porcentaje de agua máxima que puede contener una hoja a plena turgencia. Investigaciones realizadas por Kramer et al., (1974) arrojan un resultado para el CHR alrededor de $60 \%$ a $70 \%$, estando el estado hídrico de la hoja íntimamente relacionado con variables fisiológicas, tales como la turgencia de las hojas, el crecimiento, la conductancia estomática, la transpiración, la fotosíntesis y la respiración.

Entonces, partiendo de lo mencionado y buscando una solución agroecológica se propone el establecimiento de un cultivo de remolacha (Beta vulgaris L.) con el objetivo de medir su respuesta fisiológica a través del peso seco, peso fresco, peso turgente y contenido hídrico relativo en un sustrato salino, en el Centro Agroecológico Ceagro San Pablo.

\section{MATERIALES Y MÉTODOS}

\section{Diseño experimental}

El estudio se llevó a cabo en el Centro de Investigación Agroecológico San Pablo (Ceagro), ubicado en el municipio de Madrid (Cundinamarca) en donde se empleó un diseño de bloque al azar (DBCA), en una primera experiencia se hizo el tratamiento con un manejo agroecológico, y en una segunda se hizo con el método convencional, las mediciones se realizaron los días en los que se instalaron los bloques. La siembra del cultivo de Remolacha (Beta vulgaris L.) se hizo en un área de $250 \mathrm{~m} 2$, para un total de diez camas, cada una con una densidad de 210 plantas.

\section{Recolección del material vegetal}

La recolección de las muestras se realizó a los 45, 60 y 120 días respectivamente después de la siembra y se hicieron las medidas fisiológicas in vivo de peso fresco, peso turgente y peso seco para determinar el CHR (Contenido Hídrico Relativo) y analizar el estrés generado por el sustrato salino.

\section{Parámetros analizados \\ Medida del Contenido Hídrico Relativo (CHR)}

El Contenido Hídrico Relativo foliar se calculó por medio del método gravimétrico. Para ello, en primer lugar se determinó el peso fresco (PF) de las hojas tras la cosecha. Posteriormente las muestras se introdujeron en tubos con agua destilada y se refrigeraron a $4^{\circ} \mathrm{C}$; transcurridas 24 horas se anotó el peso turgente (PT). Por último, el peso seco (PS) se obtuvo a las 72 horas de introducir el material en estufa a $80^{\circ} \mathrm{C}$. (Mena-Petite et al, 1999).

El Contenido Hídrico Relativo se calculó mediante la siguiente fórmula:

$$
C H R(\%)=\frac{P F-P S}{P T-P S} * 100
$$




\section{RESULTADOS Y DISCUSIONES}

En la investigación se obtuvo un resultado del $69 \%$ de CHR para el tratamiento convencional, en tanto que en el agroecológico el resultado fue del $83 \%$, lo que indica que los tejidos se encuentran en óptimas condiciones y no son afectados por el estrés salino, esto permite afirmar, partiendo de Casseres (1981), que el cultivo Beta vulgaris es tolerante a sustratos salinos. No obstante, (Jaime et al., 2005) clasifican el cultivo halófito Beta vulgaris como tolerante a sustratos no mayores a $3 \mathrm{dS} \mathrm{m}-1$, pero en la presente investigación se pueden observar contenidos hídricos relativos por encima del $60 \%$ en un sustrato salino, el cual presenta una conductividad eléctrica de 4.2 dS m-1. Clasificado, según el Laboratorio de Salinidad de Estados Unidos (Riverside, California) como fuertemente salino.

En el tratamiento agroecológico se observó un CHR del $83 \%, 88 \%$ y $73 \%$, a los 45,60 y 120 días respectivamente, después de haber sembrado las plántulas en el campo como se evidencia en la Figura 1.

La investigación demuestra que el tratamiento agroecológico es una medida consecuente al estableci- miento del cultivo Beta vulgaris, debido a que la no presencia de trazas químicas (fertilizantes) en el ciclo fenológico del cultivo contribuyen a un volumen de agua óptimo para el desarrollo fisiológico de la planta.

Por otro lado en el tratamiento convencional se puede observar un CHR $83 \%, 69 \%$ y $71 \%$ a los 45,60 y 120 días respectivamente, lo que se evidencia en los resultados de la Figura 2.

Es evidente una disminución del CHR en el tratamiento convencional como se grafica en las figuras, puesto que la aplicación de fertilizantes aumenta la tasa de salinidad en el suelo como lo asegura en sus investigaciones (Jaime et al., 2005).

Aunque no es estadísticamente significativa la diferencia entre el tratamiento convencional y agroecológico (Figura 3), el incremento de iones se vio afectado por la aplicación de fertilizantes debido a que son sales y por lo tanto contribuyen al incremento de la conductividad eléctrica de la solución del sustrato. Sin embargo, la contribución de cloro proveniente del $\mathrm{KCl}$, en relación a los otros tipos de fertilizantes, es relativamente pequeña (Tarchitzky \& Magen, 1997).

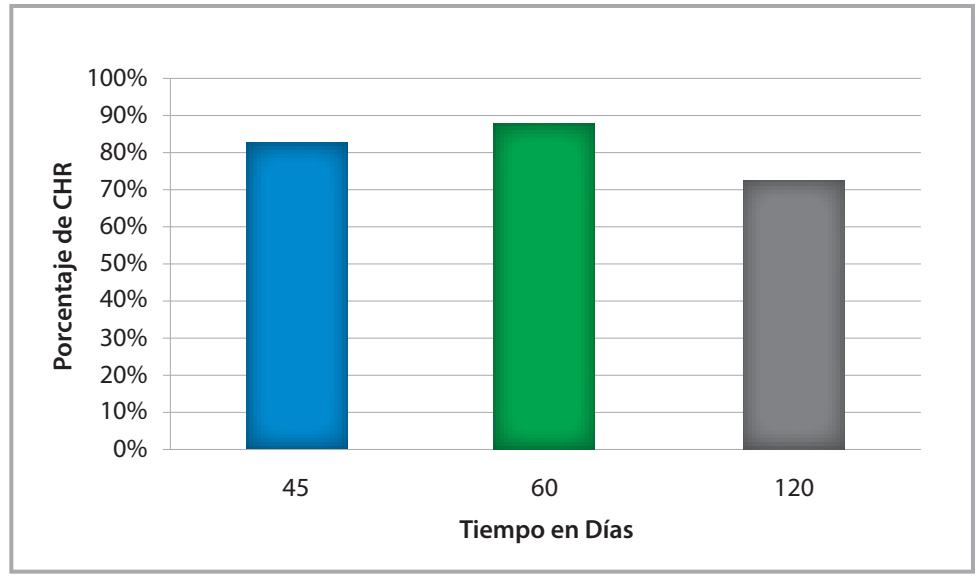

Figura 1. CHR del Cultivo de remolacha en el tratamiento Agroecológico. Por: autores, 2012.

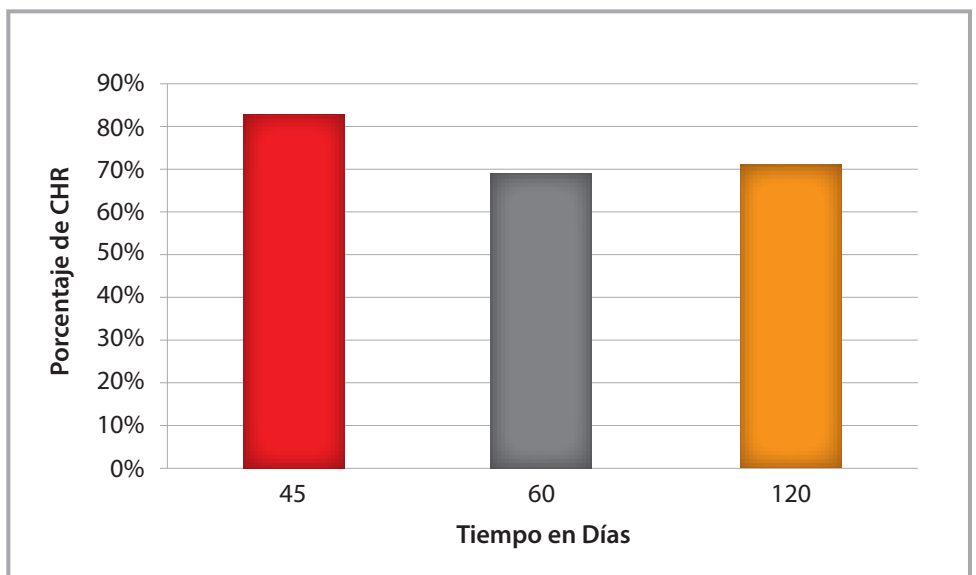

Figura 2. CHR del Cultivo de remolacha en el tratamiento convencional. Por: autores, 2012. 


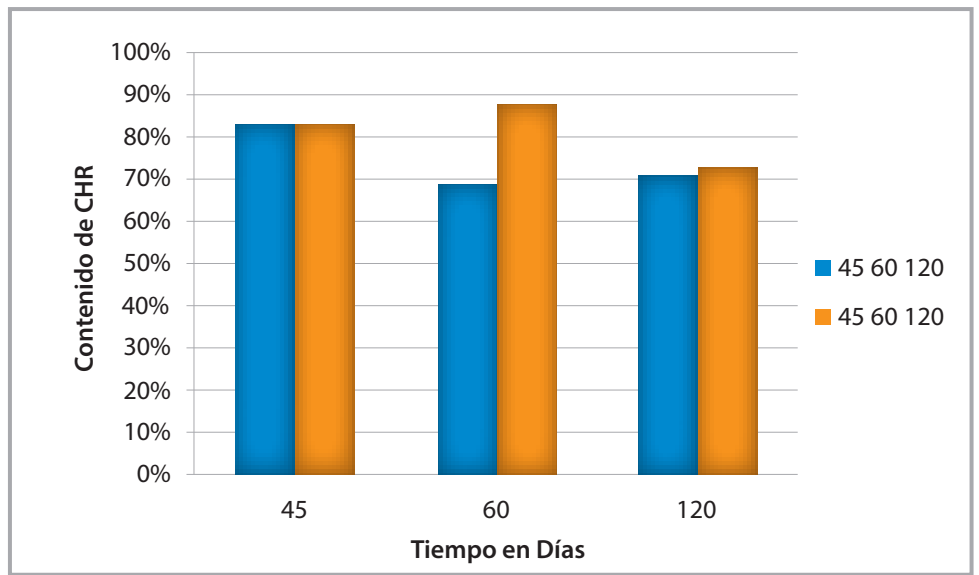

Figura 3. CHR del cultivo de remolacha en el tratamiento agroecológico y convencional. Por: autores, 2012

\section{CONCLUSIONES}

Con base en el Laboratorio de Salinidad de Estados Unidos y su clasificación para suelos salinos, se puede ubicar al Ceagro San Pablo como un suelo fuertemen-

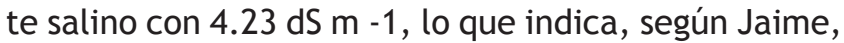
López, \& Acevedo (2005), que el cultivo no se puede establecer por problemas de toxicidad. No obstante, como se puede apreciar en la presente investigación, ambos tratamientos presentan un óptimo rango de adaptación a condiciones de salinización, en los cuales se aprecia un contenido hídrico relativo por encima del $60 \%$.

Sin duda alguna se evidenció que la aplicación de fertilizantes de síntesis química es un factor determinante para aumentar la salinización de la solución del suelo, ya que los datos arrojados por el tratamiento convencional así lo revelan, los cuales, si bien no son significativos, pueden contribuir a futuro en el deterioro de la capa cultivable.

La investigación demostró que a pesar de las condiciones edáficas del Ceagro San Pablo (alto contenido de sales), el cultivo de remolacha es tolerante a suelos salinos, pero sus condiciones fisiológicas tienen un leve cambio dependiendo del tratamiento agrotécnico empleado en el cultivo, lo que se notó en los resultados arrojados en el tratamiento agroecológico donde se evidenció un mejor contenido hídrico en cuanto a turgencia en la planta.

Esta investigación revela que tratamientos alternativos como el manejo agroecológico, aportan soluciones responsables al sistema suelo, dado que el uso de los planes de fertilización en condiciones agroecológicas es más viable en cualquier agrosistema, lo que conlleva a la conservación de los suelos, teniendo como referente el mínimo impacto ambiental.

\section{REFERENCIAS BIBLIOGRÁFICAS}

1. Ashraf, M. (1993) Effect of sodium chloride on water relations and some organic osmotica in arid zone plant species Melilotus indica (L.) All. Tropenlandwirt, 94, 95-102.

2. Campos, J. (2009) Manual de Agricultura y Ganadería Ecológica. Madrid: Mundi-prensa Libros.

3. Casseres, E. (1980) Producción de hortalizas. Venezuela : IICA Biblioteca Venezuela.

4. Florez, A.: Gálvez, V.: Hernandez, O.: López, J.: Aguirre, A. y Gallego, R. (1996) Salinidad un nuevo concepto. Colima. Mexico: UCOL.

5. Jain, R.K. y Selvaraj, G. (1997) Molecular genetic improvement of salt tolerance in plants. Biotechnology Annual Review, 3, 245-267.

6. Jaime, P.; López, M. y Acevedo, R. (2005) Agenda de campo de suelos. Madrid: Mundi-prensa Libros.

7. Kramer, P. (1974) Relaciones hídricas del suelo y plantas. Una síntesis moderna. 538.

8. Hasegawa, P.: Bressan, R. y Handa, A. (1980) Growth characteristic of $\mathrm{NaCl}$-selected and non-selected cells of Nicotiana tabacum $L$. Plant and Cell Physiology, 21, 1347-1355.

9. Hasegawa, P.; Bressan, R.; Zhu J.K. y Bohnert, H.J. (2000) Plant cellular and molecular responses to high salinity. Annual Review of Plant Physiology and Plant Molecular Biology, 51: 463-499. 
10. Mena-Petite, A.; Duñabeitia, M.K.; González-Moro, B.; Muñoz-Rueda, A. y Lacuesta, M.; (1999) Sequential effects of acidic precipitation and drought on water relations of Pinus radiata seedlings. J. Plant Physiol. 155: 93-100.

11. Ortiz, E., Leidi, y Pardo, J. (2002) Tolerancia de los cultivos al estrés salino: qué hay de nuevo. Revista de Investigaciones de la Facultad de Ciencias Agrarias Universidad Nacional de Rosario, II, 2.

12. Royo, A. y Cantero, C. (1996) Efecto de la salinidad sobre la cebada (Hordeum vulgare L.). Análisis de caracteres morfo-fisiológicos y su relación con la tolerancia a la salinidad. (Tesis de Master). España: Universidad de Lérida
13. Shannon, M. (1997) Adaptation of plants to salinity. Advances in Agronomy, 60, 75-120.

14. Tarchitzky, J. y Magen. (mayo, 1997) Status of potassium in soils and crops in Israel, present $K$ use indicating the need for further research and improved recommendations. Presentado en Taller Regional IPI sobre Seguridad Alimentaria en la Región de Wanna. Bornova, Turquía.

15. Yeo, A.R. (1998). Molecular biology of salt tolerance in the context of whole-plant physiology. Journal of Experimental Botany, 49, 915-929. 